\title{
Accuracy of spin-component scaled ADC(2) excitation energies and potential energy surfaces
}

\author{
Attila Tajti, ${ }^{*}$ Levente Tulipán, and Péter G. Szalay* \\ ELTE Eötvös Loránd University, Institute of Chemistry, Laboratory of Theoretical \\ Chemistry, , P. O. Box 32, H-1518, Budapest 112, Hungary \\ E-mail: tat@chem.elte.hu; szalay@chem.elte.hu
}




\begin{abstract}
In a recent paper of this Journal (Tajti and Szalay, JCTC 2019, 15, 5523) we have shown that failures of the CC2 method to describe Rydberg excited states, as well as potential energy surfaces of certain valence excited states can be cured by spincomponent scaled (SCS) versions SCS-CC2 and SOS-CC2 by a large extent. In this paper, the related and popular Second Order Algebraic Diagrammatic Construction $(\mathrm{ADC}(2))$ method and its SCS variants are inspected with the previously established methodology. The results reflect the similarity of the $\mathrm{CC} 2$ and $\mathrm{ADC}(2)$ models, showing identical problems in the case of the canonical form and the same improvement when spin-component-scaling is applied.
\end{abstract}

\title{
1 Introduction
}

In two recent papers ${ }^{1,2}$ in this Journal, we have extended the large pool of benchmark calculations on vertical excitation energy ${ }^{3-14}$ with characterisation of the excited state potential energy surfaces using Coupled-Cluster (CC) type methods. In the first paper we have found ${ }^{1}$ that the popular $\mathrm{CC} 2$ method $^{15}$ fails sometimes badly, depending on the type of the excited state. The parent CCSD method (termed either EOM-CCSD ${ }^{16-18}$ or CCSD-LR ${ }^{19,20}$ ) gives usually reasonable results, but the inclusion of approximate triple excitations is necessary to achieve high accuracy. Since this is generally not possible for larger systems, improved second order approximations are requested. ${ }^{12,13}$ Along this line, in our recent study ${ }^{2}$ we also investigated spin-component scaled versions of $\mathrm{CC} 2^{21,22}$ and found that both the scaledopposite-spin (SOS-CC2) and spin-component-scaled (SCS-CC2) variants cure the problem of $\mathrm{CC} 2$ to a considerable extent and the resulting potential energy surfaces run quite close to those obtained with CCSD.

Besides $\mathrm{CC} 2$, another second order method often used in applications is the so called ADC(2) (2nd order Algebraic Diagrammatic Construction) method. ${ }^{23,24}$ This method is closely related to $\mathrm{CC} 2,{ }^{25}$ and it is a widespread experience that $\mathrm{CC} 2$ and $\mathrm{ADC}(2)$ results 
are of very similar quality. ${ }^{7,25-27}$

In this paper we augment the results of our previous paper ${ }^{2}$ and present the results of the same type of calculations, now using the $\mathrm{ADC}(2)$ and its spin-component scaled variants SOS-ADC(2) and SCS-ADC(2). We believe that this addition is necessary to get a complete picture about the performance of the second order methods which can be used and are being used to describe excited states of larger molecules.

\section{Methods}

\subsection{The $\mathrm{ADC}(2)$ method}

The 2nd order Algebraic Diagrammatic Construction $(\mathrm{ADC}(2))$ method has been introduced by Schirmer, ${ }^{23,28}$ based on a diagrammatic perturbational expansion of the polarization propagator. ${ }^{23}$ Later the $\mathrm{ADC}(2)$ method has been reintroduced by Trofimov and Schirmer. ${ }^{24,29}$ The real advent of the method came when its relation to CC2 has been established and its implementation in program codes, e.g. in TURBOMOLE ${ }^{30}$ pursued. The close relation of CC2 and $\mathrm{ADC}(2)$ has been discussed by Hättig ${ }^{25}$ and recently in detail by Izsák, ${ }^{27}$ showing that $\mathrm{ADC}(2)$ can be considered as an approximation to $\mathrm{CC} 2$ by neglecting $\mathrm{T}_{1}$ amplitudes (thus enforcing strictly second order) and symmetrising the Jacobian. As has been shown e.g. in ref. 12 , neglect of $\mathrm{T}_{1}$ amplitudes has a negligible effect on excitation energies. Symmetrisation, on the other hand, avoids technical issues related to the non-Hermitian formulation. ${ }^{25}$ These two approximations also result in a slightly lower cost.

\subsection{Spin-component scaled $\operatorname{ADC}(2)$}

The concept of spin-component scaled $\mathrm{CC} 2$, i.e. the introduction of spin scaling factors $\mathrm{C}_{O S}$ and $\mathrm{C}_{S S}$ into the formulae of the effective Hamiltonian has been first established by Hättig et al., ${ }^{21}$ in the spirit of spin-component-scaled MP2 originally proposed by Grimme. ${ }^{31,32}$ In case of the SCS (spin-component-scaled) variant the same-spin integrals are multiplied by 
a factor of $\mathrm{C}_{S S}=1 / 3$, while the opposite-spin integrals by $\mathrm{C}_{O S}=6 / 5$. In the SOS (scaledopposite-spin) version only the opposite-spin integrals are retained $\left(\mathrm{C}_{O S}=1.3, \mathrm{C}_{S S}=0.0\right)$. Using the above mentioned similarity of $\mathrm{CC} 2$ and $\mathrm{ADC}(2)$ theories, the same spin-scaled modifications can be introduced into $\operatorname{ADC}(2)$ in a straightforward manner, leading to the SCS-ADC(2) and SOS-ADC(2) models. The latter was first realized by Winter and Hättig, ${ }^{22}$ and later by Dreuw and co-workers ${ }^{7,33}$ and, recently, by Mester and co-workers. ${ }^{34,35}$ Today, implementations are available in the TURBOMOLE ${ }^{30}$ and $\mathrm{MRCC}^{36}$ program packages. The particular choice of SOS-ADC(2) is of outstanding importance for applications on large systems as, if combined with the Resolution-of-Identity approximation of the two-electron

integrals, ${ }^{37}$ it can be formulated to obey a computational cost scaling with no more than the fourth power of the system size. ${ }^{22,38}$

\subsection{Computational details}

The same methodology as in the parent paper ${ }^{2}$ has been used. To benchmark the accuracy of vertical excitation energies, 64 valence and 56 Rydberg states of 23 molecules have been calculated using the aug-cc-pVDZ basis set, with the core electrons not correlated. The calculations were performed using the TURBOMOLE ${ }^{30}$ program system. As reference, the CC3-LR excitation energies obtained in ref. 12 are used.

The characterisation of the potential energy surfaces has been performed for the low-lying valence excited states of methanimine, formamide, cytosine and guanine, explored previously in refs. 1 and 2. As a reference, CCSDT results from ref. 1 were used, except for guanine, where the reference values are calculated ${ }^{1}$ by the $\operatorname{EOM-CCSD}(T)(a) *$ method of Matthews and Stanton. ${ }^{39}$ This choice has been justified in ref. 1 showing that EOM-CCSD $(\mathrm{T})(\mathrm{a})^{*}$ results are very close to CCSDT ones.

Gradients of the vertical excitation energy at the Franck-Condon point have been calculated numerically using the $\mathrm{MRCC}^{36}$ program in the cc-pVDZ basis set. For comparison of the gradients, the length of the vectors, as well as their relative angle with respect to the 
reference values is evaluated.

Finally, the surface plots that follow the Frank-Condon forces in mass-weighted steps (for a detailed definition, see our previous paper ${ }^{1}$ ) have also been evaluated in the cc-pVDZ basis by the MRCC program. ${ }^{36}$

\section{Results and discussion}

\subsection{Vertical excitation energies}

Statistics on the error of the vertical excitation energies are presented in table 1 for $\operatorname{ADC}(2)$ and its spin-component scaled variants, evaluated against CC3 reference values. The respective CC2, SCS-CC2, SOS-CC2, and CCSD results from ref. 2 are shown for comparison. (The full set of results for the excitation energies is available in the Supporting Information.) The errors of $\mathrm{ADC}(2)$ lie close to those of the unscaled CC2 model. In particular, the severe underestimation of Rydberg excitation energies is very apparent, with an error of $-0.42 \mathrm{eV}$ being worse than even CC2 by $0.04 \mathrm{eV}$. Nevertheless, the valence-Rydberg misbalance, shown in the last column of table 1 , is exactly as large as that of CC2. The two spin-component scaled $\mathrm{ADC}(2)$ variants show statistics very similar to their CC2 counterparts, the difference of the mean errors being no more than $0.03 \mathrm{eV}$, and even the standard deviations follow the same pattern. The above mentioned misbalance is reduced by spin-component scaling by as much as 38 and 55 percent for the SCS-ADC(2) and SOS-ADC(2) models, respectively, essentially identical to the SCS-CC2 and SOS-CC2 results.

\subsection{Franck-Condon gradients}

Measures on the gradients of the excited state energies at the ground state equilibrium geometry are presented in tables $2,3,4$, and 5 . The $\mathrm{ADC}(2)$ gradients, as evaluated by their length and their direction relative to the reference vectors, show an accuracy similar to that of $\mathrm{CC} 2$, with no clear indication of one being better than the other. Compared to CC2, in many 
cases a larger error of the gradient length is accompanied by a more accurate direction of the vector, or vice versa. However, in situations where the description is clearly problematic (e.g. in some $n-\pi^{*}$ states of nucleobases), both measures are inferior to the CC2 result. For such states, the effect of spin-component scaling is generally also similar, marginally inferior compared to the respective $\mathrm{CC} 2$ variant, i.e. both $\mathrm{SCS}-\mathrm{ADC}(2)$ and $\mathrm{SOS}-\mathrm{ADC}(2)$ provide significantly better gradients compared to the canonical model. The only exception seems to be the $\pi-\pi^{*}$ state of formamide, where no improvement is seen for the ADC(2) methods, while the CC2 result is clearly surpassed by the spin-component scaled versions. This can be, however, attributed to the fact that the $\mathrm{CC} 2$ and $\mathrm{ADC}(2)$ single excitation vectors of this state show a slightly different mixing of dominant $\pi-\pi^{*}$ contributions. For excited states where the CC2 gradient is found to be satisfactory, the spin-component scaling in $\mathrm{ADC}(2)$ shifts the canonical values in the very same direction as seen in the CC2 cases. Although this does not clearly lead to more accurate gradients, the differences remain negligible as the nominal errors remain low in all such cases.

\subsection{Surface scans}

The excited state potential energy surface scans shown on figures 1, 2, 3, and 4 generally seem to emphasize the above findings even further. The $\mathrm{ADC}(2)$ and $\mathrm{CC} 2$ curves run very close to each other, at least in the first half of the explored PES. That is, wherever $\mathrm{ADC}(2)$ and $\mathrm{CC} 2$ gradients were found to be exceptionally inaccurate (like in case of the first $n-\pi^{*}$ state of cytosine), the respective plots form a well recognizable, distinct pair. The spin-component scaled $\mathrm{ADC}(2)$ models show a pattern similar to the one found earlier for their CC2 counterparts: ${ }^{2}$ their divergence from the reference curve, as compared to regular $\mathrm{ADC}(2)$, is reduced considerably, giving nearly CCSD quality results for most investigated states. SOS-ADC(2) performs slightly better than SCS-ADC(2) in this analysis, but the difference of the two variants remains minor compared to how much they both improve over spin-unscaled $\operatorname{ADC}(2)$. 


\section{Conclusions}

The results presented above affirm earlier conclusions that $\mathrm{ADC}(2)$ and $\mathrm{CC} 2$ are closely related methods: not only statistics on vertical excitation energies for both valence and Rydberg states are close, but also the Frank-Condon gradients show very similar patterns. In case of the surface scans one sees a failure of $\mathrm{ADC}(2)$ in situations when $\mathrm{CC} 2$ fails, as well. Noteworthy differences happen only for near-degeneracies where the character of the single excitation part of the solution vectors might be different for the two methods. Scaling spin-components differently in $\mathrm{ADC}(2)$ brings a significant improvement in most cases, very similarly to the CC2 case. Both the systematic error of the Rydberg excitation energies, and the misbalance between valence and Rydberg states are reduced. It also spectacularly corrects the potential energy surfaces when $\mathrm{ADC}(2)$ fails, while conserving the accuracy if it does not.

Therefore SOS-ADC(2), along with SOS-CC2, seems to be an economic and accurate variant for large calculations, considering the lowest, fourth power scaling of these methods with the system size. One should keep in mind, however, that spin-component scaling is mostly empirical (for an approximate theoretical justification for ground state see the paper of Szabados ${ }^{40}$ ), and therefore the good performance can not be guaranteed for all systems and all situations.

\section{Acknowledgement}

This work has been supported by the National Research, Innovation and Development Fund (NKFIA) Grant No. 124293.

\section{Supporting Information Available}


Table 1: Statistics on relative vertical excitation energies compared to CC3-LR, in electron volts

\begin{tabular}{|c|c|c|c|c|c|c|c|}
\hline \multirow{2}{*}{ Method } & \multicolumn{3}{|c|}{ Valence States } & \multicolumn{3}{|c|}{ Rydberg States } & \multirow{2}{*}{ Mean $\Delta$ (Valence -Rydberg) } \\
\hline & Mean $\Delta \mathrm{E}$ & $\sigma_{\triangle \mathrm{F}}$ & $\operatorname{Max} .|\Delta \mathrm{E}|$ & Mean $\Delta \mathrm{E}$ & $\sigma_{\triangle \mathrm{F}}$ & $\operatorname{Max} .|\Delta \mathrm{E}|$ & \\
\hline $\mathrm{CC} 2^{1}$ & 0.02 & 0.10 & 0.40 & -0.38 & 0.26 & 0.68 & 0.40 \\
\hline $\operatorname{ADC}(2)$ & -0.03 & 0.12 & 0.38 & -0.42 & 0.30 & 0.81 & 0.40 \\
\hline SCS-CC2 & 0.18 & 0.14 & 0.53 & -0.07 & 0.14 & 0.48 & 0.24 \\
\hline SCS-ADC(2) & 0.17 & 0.16 & 0.87 & -0.08 & 0.18 & 0.59 & 0.25 \\
\hline SOS-CC2 & 0.26 & 0.20 & 0.71 & 0.08 & 0.16 & 0.56 & 0.18 \\
\hline SOS-ADC $(2)$ & 0.23 & 0.19 & 0.65 & 0.05 & 0.17 & 0.53 & 0.18 \\
\hline EOM-CCSD & 0.17 & 0.13 & 0.42 & 0.11 & 0.08 & 0.33 & 0.07 \\
\hline
\end{tabular}


Table 2: Vertical excitation energies (in electron volts), as well as the length (in atomic units) and angle with respect to CCSDT (degrees) of excited state gradient vectors of Methanimine

\begin{tabular}{|c|c|c|c|c|c|c|c|}
\hline & $\begin{array}{l}E_{e x c}^{i} \\
(\mathrm{eV})\end{array}$ & $\begin{array}{l}\left\|\bar{\nabla} E^{i}\right\| \\
\text { (a.u.) }\end{array}$ & $\begin{array}{l}\measuredangle_{\mathrm{CCSDT}} \\
\text { (deg.) }\end{array}$ & & $\begin{array}{l}E_{e x c}^{i} \\
(\mathrm{eV})\end{array}$ & $\begin{array}{l}\left\|\bar{\nabla} E^{i}\right\| \\
\text { (a.u.) }\end{array}$ & $\begin{array}{l}\measuredangle_{\text {CCSDT }} \\
\text { (deg.) }\end{array}$ \\
\hline \multicolumn{8}{|c|}{ State $1\left(n-\pi^{*}\right)$} \\
\hline SCS-CC2 & 5.73 & 0.1636 & 1.56 & SCS-ADC(2) & 5.69 & 0.1631 & 1.62 \\
\hline SOS-CC2 & 5.78 & 0.1641 & 1.58 & SOS-ADC (2) & 5.73 & 0.1637 & 1.63 \\
\hline $\mathrm{CC} 2$ & 5.65 & 0.1626 & 1.56 & $\operatorname{ADC}(2)$ & 5.61 & 0.1619 & 1.68 \\
\hline CCSD & 5.54 & 0.1576 & 4.37 & CCSDT & 5.47 & 0.1692 & 0.00 \\
\hline \multicolumn{8}{|c|}{ State $2\left(\sigma-\pi^{*}\right)$} \\
\hline SCS-CC2 & 10.01 & 0.2543 & 6.37 & SCS-ADC (2) & 10.01 & 0.2444 & 8.10 \\
\hline SOS-CC2 & 10.05 & 0.2518 & 6.50 & SOS-ADC $(2)$ & 10.06 & 0.2407 & 8.65 \\
\hline $\mathrm{CC} 2$ & 9.92 & 0.2606 & 6.12 & $\operatorname{ADC}(2)$ & 9.93 & 0.2516 & 7.36 \\
\hline CCSD & 9.79 & 0.2423 & 3.18 & CCSDT & 9.65 & 0.2387 & 0.00 \\
\hline
\end{tabular}

\section{References}

(1) Tajti, A.; Stanton, J. F.; Matthews, D. A.; Szalay, P. G. Accuracy of Coupled Cluster Excited State Potential Energy Surfaces. J. Chem. Theory Comput. 2018, 14, 58595869.

(2) Tajti, A.; Szalay, P. G. Accuracy of Spin-Component-Scaled CC2 Excitation Energies and Potential Energy Surfaces. Journal of Chemical Theory and Computation 2019, 15, 5523, PMID: 31433639.

(3) Schreiber, M.; Silva, M. R. J.; Sauer, S. P. A.; Thiel, W. Benchmarks for electronically excited states: CASPT2, CC2, CCSD, and CC3. The Journal of Chemical Physics 2008, 128, 134110 .

(4) Sauer, S. P. A.; Schreiber, M.; Silva-Junior, M. R.; Thiel, W. Benchmarks for Electronically Excited States: A Comparison of Noniterative and Iterative Triples Corrections in 
Table 3: Vertical excitation energies (in electron volts), as well as the length (in atomic units) and angle with respect to CCSDT (degrees) of excited state gradient vectors of Formamide

\begin{tabular}{|c|c|c|c|c|c|c|c|}
\hline & $\begin{array}{l}E_{e x c}^{i} \\
(\mathrm{eV})\end{array}$ & $\begin{array}{l}\left\|\bar{\nabla} E^{i}\right\| \\
\text { (a.u.) }\end{array}$ & $\begin{array}{l}\measuredangle_{\mathrm{CCSDT}} \\
\text { (deg.) }\end{array}$ & & $\begin{array}{l}E_{e x c}^{i} \\
(\mathrm{eV})\end{array}$ & $\begin{array}{l}\left\|\bar{\nabla} E^{i}\right\| \\
\text { (a.u.) }\end{array}$ & $\begin{array}{l}\measuredangle_{\mathrm{CCSDT}} \\
\text { (deg.) }\end{array}$ \\
\hline \multicolumn{8}{|c|}{ State $1\left(n-\pi^{*}\right)$} \\
\hline SCS-CC2 & 6.05 & 0.3010 & 4.90 & $\operatorname{SCS}-\operatorname{ADC}(2)$ & 5.78 & 0.3129 & 2.26 \\
\hline SOS-CC2 & 6.03 & 0.2986 & 4.75 & SOS-ADC $(2)$ & 5.81 & 0.3099 & 1.73 \\
\hline $\mathrm{CC} 2$ & 6.00 & 0.3061 & 5.26 & $\operatorname{ADC}(2)$ & 5.73 & 0.3190 & 3.31 \\
\hline CCSD & 5.87 & 0.2678 & 1.13 & CCSDT & 5.84 & 0.2850 & 0.00 \\
\hline \multicolumn{8}{|c|}{ State $2\left(\pi-\pi^{*}\right)$} \\
\hline SCS-CC2 & 8.05 & 0.2196 & 8.07 & $\operatorname{SCS}-\mathrm{ADC}(2)$ & 7.81 & 0.2365 & 11.17 \\
\hline SOS-CC2 & 8.01 & 0.2265 & 15.92 & SOS-ADC $(2)$ & 7.87 & 0.2465 & 16.29 \\
\hline $\mathrm{CC} 2$ & 7.71 & 0.2370 & 16.00 & $\operatorname{ADC}(2)$ & 7.63 & 0.2367 & 13.72 \\
\hline CCSD & 7.92 & 0.1975 & 6.87 & CCSDT & 7.64 & 0.2066 & 0.00 \\
\hline
\end{tabular}

Table 4: Vertical excitation energies (in electron volts), as well as the length (in atomic units) and angle with respect to CCSDT (degrees) of excited state gradient vectors of Cytosine

\begin{tabular}{|c|c|c|c|c|c|c|c|}
\hline & $\begin{array}{l}E_{e x c}^{i} \\
(\mathrm{eV})\end{array}$ & $\begin{array}{l}\left\|\bar{\nabla} E^{i}\right\| \\
\text { (a.u.) }\end{array}$ & $\begin{array}{l}\measuredangle_{\mathrm{CCSDT}} \\
\text { (deg.) }\end{array}$ & & $\begin{array}{l}E_{e x c}^{i} \\
(\mathrm{eV})\end{array}$ & $\begin{array}{l}\left\|\bar{\nabla} E^{i}\right\| \\
\text { (a.u.) }\end{array}$ & $\begin{array}{l}\measuredangle_{\mathrm{CCSDT}} \\
\text { (deg.) }\end{array}$ \\
\hline \multicolumn{8}{|c|}{ State $1\left(\pi-\pi^{*}\right)$} \\
\hline SCS-CC2 & 5.06 & 0.2354 & 3.18 & SCS-ADC(2) & 4.90 & 0.2403 & 3.88 \\
\hline SOS-CC2 & 5.10 & 0.2316 & 5.51 & SOS-ADC (2) & 4.96 & 0.2351 & 5.83 \\
\hline $\mathrm{CC} 2$ & 4.96 & 0.2467 & 4.44 & $\mathrm{ADC}(2)$ & 4.77 & 0.2541 & 5.15 \\
\hline CCSD & 5.11 & 0.2034 & 8.08 & CCSDT & 4.86 & 0.2267 & 0.00 \\
\hline \multicolumn{8}{|c|}{ State $2\left(\pi-\pi^{*}\right)$} \\
\hline SCS-CC2 & 6.05 & 0.2203 & 8.73 & SCS-ADC(2) & 5.91 & 0.2169 & 8.93 \\
\hline SOS-CC2 & 6.14 & 0.2316 & 10.36 & SOS-ADC (2) & 6.00 & 0.2174 & 10.70 \\
\hline $\mathrm{CC} 2$ & 5.86 & 0.2171 & 7.24 & $\mathrm{ADC}(2)$ & 5.71 & 0.2125 & 6.72 \\
\hline CCSD & 6.10 & 0.1961 & 7.73 & CCSDT & 5.75 & 0.2095 & 0.00 \\
\hline \multicolumn{8}{|c|}{ State $3\left(n-\pi^{*}\right)$} \\
\hline SCS-CC2 & 5.52 & 0.1706 & 5.83 & SCS-ADC $(2)$ & 5.37 & 0.1747 & 6.26 \\
\hline SOS-CC2 & 5.65 & 0.1645 & 14.13 & SOS-ADC (2) & 5.52 & 0.1665 & 14.28 \\
\hline $\mathrm{CC} 2$ & 5.15 & 0.2489 & 35.46 & $\operatorname{ADC}(2)$ & 4.96 & 0.2622 & 37.04 \\
\hline CCSD & 5.53 & 0.1538 & 21.45 & CCSDT & 5.28 & 0.1628 & 0.00 \\
\hline
\end{tabular}


Table 5: Vertical excitation energies (in electron volts), as well as the length (in atomic units) and angle with respect to $\operatorname{CCSD}(\mathrm{T})(\mathrm{a}) *$ (degrees) of excited state gradient vectors of Guanine

\begin{tabular}{|c|c|c|c|c|c|c|c|}
\hline & $\begin{array}{l}E_{e x c}^{i} \\
(\mathrm{eV})\end{array}$ & $\begin{array}{l}\left\|\bar{\nabla} E^{i}\right\| \\
\text { (a.u.) }\end{array}$ & $\begin{array}{c}\measuredangle_{\mathrm{CCSD}(\mathrm{T})(\mathrm{a})^{*}} \\
\text { (deg.) }\end{array}$ & & $\begin{array}{l}E_{e x c}^{i} \\
(\mathrm{eV})\end{array}$ & $\begin{array}{l}\left\|\bar{\nabla} E^{i}\right\| \\
\text { (a.u.) }\end{array}$ & $\begin{array}{c}\measuredangle_{\mathrm{CCSD}(\mathrm{T})(\mathrm{a})^{*}} \\
(\mathrm{deg} .)\end{array}$ \\
\hline \multicolumn{8}{|c|}{ State $1\left(\pi-\pi^{*}\right)$} \\
\hline SCS-CC2 & 5.38 & 0.1947 & 4.08 & SCS-ADC(2) & 5.28 & 0.1961 & 4.61 \\
\hline SOS-CC2 & 5.40 & 0.1938 & 3.91 & SOS-ADC(2) & 5.30 & 0.1939 & 4.65 \\
\hline $\mathrm{CC} 2$ & 5.35 & 0.1982 & 6.39 & $\operatorname{ADC}(2)$ & 5.22 & 0.2013 & 5.60 \\
\hline CCSD & 5.80 & 0.1697 & 6.60 & $\operatorname{CCSD}(\mathrm{T})(\mathrm{a})^{*}$ & 5.75 & 0.1843 & 0.00 \\
\hline \multicolumn{8}{|c|}{ State $2\left(\pi-\pi^{*}\right)$} \\
\hline SCS-CC2 & 6.03 & 0.2062 & 3.98 & SCS-ADC (2) & 5.99 & 0.2039 & 5.38 \\
\hline SOS-CC2 & 6.11 & 0.2070 & 5.10 & SOS-ADC(2) & 6.06 & 0.2046 & 5.05 \\
\hline $\mathrm{CC} 2$ & 5.88 & 0.2077 & 4.94 & $\operatorname{ADC}(2)$ & 5.82 & 0.2025 & 7.93 \\
\hline CCSD & 6.11 & 0.1798 & 6.60 & $\operatorname{CCSD}(\mathrm{T})(\mathrm{a})^{*}$ & 5.91 & 0.1957 & 0.00 \\
\hline \multicolumn{8}{|c|}{ State $3\left(\pi-\pi^{*}\right)$} \\
\hline SCS-CC2 & 7.02 & 0.1771 & 5.88 & SCS-ADC(2) & 6.98 & 0.1632 & 6.41 \\
\hline SOS-CC2 & 7.07 & 0.1732 & 7.21 & SOS-ADC (2) & 7.02 & 0.1586 & 7.42 \\
\hline $\mathrm{CC} 2$ & 6.91 & 0.1843 & 6.57 & $\mathrm{ADC}(2)$ & 6.88 & 0.1708 & 6.99 \\
\hline CCSD & 7.10 & 0.1498 & 6.04 & $\operatorname{CCSD}(\mathrm{T})(\mathrm{a})^{*}$ & 6.87 & 0.1788 & 0.00 \\
\hline \multicolumn{8}{|c|}{ State $4\left(n-\pi^{*}\right)$} \\
\hline SCS-CC2 & 5.87 & 0.3024 & 1.63 & SCS-ADC(2) & 5.64 & 0.3165 & 3.12 \\
\hline SOS-CC2 & 5.92 & 0.2979 & 2.55 & SOS-ADC(2) & 5.70 & 0.3112 & 3.93 \\
\hline $\mathrm{CC} 2$ & 5.75 & 0.3173 & 5.21 & $\operatorname{ADC}(2)$ & 5.50 & 0.3284 & 4.42 \\
\hline CCSD & 5.80 & 0.2626 & 4.94 & $\operatorname{CCSD}(T)(a)^{*}$ & 5.75 & 0.2835 & 0.00 \\
\hline \multicolumn{8}{|c|}{ State $5\left(n-\pi^{*}\right)$} \\
\hline SCS-CC2 & 6.84 & 0.1782 & 22.20 & SCS-ADC(2) & 6.77 & 0.1749 & 29.15 \\
\hline SOS-CC2 & 6.94 & 0.1699 & 15.70 & SOS-ADC(2) & 6.88 & 0.1647 & 23.60 \\
\hline $\mathrm{CC} 2$ & 6.57 & 0.2339 & 38.52 & $\operatorname{ADC}(2)$ & 6.46 & 0.2634 & 48.92 \\
\hline CCSD & 6.87 & 0.1705 & 11.90 & $\operatorname{CCSD}(\mathrm{T})(\mathrm{a})^{*}$ & 6.73 & 0.1823 & 0.00 \\
\hline
\end{tabular}


Linear Response Coupled Cluster Methods: CCSDR(3) versus CC3. J. Chem. Theory Comput. 2009, 5, 555-564.

(5) Silva-Junior, M. R.; Sauer, S. P. A.; Schreiber, M.; Thiel, W. Basis set effects on coupled cluster benchmarks of electronically excited states: CC3, CCSDR (3) and CC2. Mol. Phys. 2010, 108, 453-465.

(6) Sous, J.; Goel, P.; Nooijen, M. Similarity transformed equation of motion coupled cluster theory revisited: a benchmark study of valence excited states. Mol. Phys. 2014, 112, 616-638.

(7) Harbach, P. H. P.; Wormit, M.; Dreuw, A. The third-order algebraic diagrammatic construction method $(\mathrm{ADC}(3))$ for the polarization propagator for closed-shell molecules: Efficient implementation and benchmarking. J. Chem. Phys. 2014, 141, 064113.

(8) Kánnár, D.; Szalay, P. G. Benchmarking Coupled Cluster Methods on Valence Singlet Excited States. J. Chem. Theory Comput. 2014, 10, 3757-3765.

(9) Jacquemin, D.; Duchemin, I.; Blase, X. 0-0 Energies Using Hybrid Schemes: Benchmarks of TD-DFT, CIS(D), ADC(2), CC2, and BSE/ GWformalisms for 80 Real-Life Compounds. J. Chem. Theory Comput. 2015, 11, 5340-5359.

(10) Piecuch, P.; Hansen, J. A.; Ajala, A. O. Benchmarking the completely renormalised equation-of-motion coupled-cluster approaches for vertical excitation energies. Mol. Phys. 2015, 113, 3085-3127.

(11) Huntington, L. M. J.; Demel, O.; Nooijen, M. Benchmark Applications of Variations of Multireference Equation of Motion Coupled-Cluster Theory. J. Chem. Theory Comput. 2016, 12, 114-132.

(12) Tajti, A.; Szalay, P. G. Investigation of the Impact of Different Terms in the Second 
Order Hamiltonian on Excitation Energies of Valence and Rydberg States. J. Chem. Theory Comput. 2016, 12, 5477-5482.

(13) Rishi, V.; Perera, A.; Nooijen, M.; Bartlett, R. J. Excited states from modified coupled cluster methods: Are they any better than EOM CCSD? J. Chem. Phys. 2017, 146, 144104.

(14) Kánnár, D.; Tajti, A.; Szalay, P. G. Accuracy of Coupled Cluster excitation energies in diffuse basis sets. J. Chem. Theory Comput. 2017, 13, 202-209.

(15) Christiansen, O.; Koch, H.; Jørgensen, P. The Second-order Approximate CoupledCluster Singles and Doubles Model CC2. Chem. Phys. Lett. 1995, 243, 409-418.

(16) Sekino, H.; Bartlett, R. J. A Linear Response, Coupled-Cluster Theory for ExcitationEnergy. Int. J. Quantum Chem. 1984, 26, 255-265.

(17) Stanton, J. F.; Bartlett, R. J. The Equation of Motion Coupled-Cluster Method A Systematic Biorthogonal Approach to Molecular-Excitation Energies, TransitionProbabilities, and Excited-State Properties. J. Chem. Phys. 1993, 98, 7029-7039.

(18) Comeau, D. C.; Bartlett, R. J. The Equation-of-Motion Coupled-Cluster Method Applications to Open-Shell and Closed-Shell Reference States. Chem. Phys. Lett. 1993, $20 \%, 414-423$.

(19) Koch, H.; Jørgensen, P. Coupled cluster response functions. J. Chem. Phys. 1990, 93, 3333.

(20) Koch, H.; Jensen, H. J. A.; Jørgensen, P.; Helgaker, T. Excitation energies from the coupled cluster singles and doubles linear response function (CCSDLR). Applications to Be, CH+, CO, and H2O. J. Chem. Phys. 1990, 93, 3345-3350.

(21) Hellweg, A.; Grün, S. A.; Hättig, C. Benchmarking the performance of spin-component 
scaled CC2 in ground and electronically excited states. Phys. Chem. Chem. Phys. 2008, 10, 4119-4127.

(22) Winter, N. O. C.; Hättig, C. Scaled opposite-spin CC2 for ground and excited states with fourth order scaling computational costs. J. Chem. Phys. 2011, 134, 184101.

(23) Schirmer, J. Beyond the Random-Phase Approximation - A New Approximation Scheme for the Polarization Propagator. Phys. Rev. A 1982, 26, 2395-2416.

(24) Trofimov, A. B.; Schirmer, J. An Efficient Polarization Propagator Approach to Valence Electron-Excitation Spectra. J. Phys. B: At. Mol. Opt. 1995, 28, 2299-2324.

(25) Hättig, C. In Response Theory and Molecular Properties (A Tribute to Jan Linderberg and Poul Jørgensen); Jensen, H., Ed.; Advances in Quantum Chemistry; Academic Press, 2005; Vol. 50; pp $37-60$.

(26) Wormit, M.; Rehn, D. R.; Harbach, P. H.; Wenzel, J.; Krauter, C. M.; Epifanovsky, E.; Dreuw, A. Investigating excited electronic states using the algebraic diagrammatic construction (ADC) approach of the polarisation propagator. Molecular Physics 2014, 112, 774-784.

(27) Izsák, R. Single-reference coupled cluster methods for computing excitation energies in large molecules: The efficiency and accuracy of approximations. Wiley Interdisciplinary Reviews: Computational Molecular Science 2019, e1445.

(28) Schirmer, J. Closed-form intermediate representations of many-body propagators and resolvent matrices. Phys. Rev. A 1991, 43, 4647-4659.

(29) Schirmer, J.; Trofimov, A. B. Intermediate state representation approach to physical properties of electronically excited molecules. The Journal of Chemical Physics 2004, 120, 11449-11464. 
(30) TURBOMOLE V7.3 2018, a development of University of Karlsruhe and Forschungszentrum Karlsruhe GmbH, 1989-2007, TURBOMOLE GmbH, since 2007; available from http://www.turbomole.com.

(31) Grimme, S. Improved second-order Møller-Plesset perturbation theory by separate scaling of parallel- and antiparallel-spin pair correlation energies. J. Chem. Phys. 2003, 118, 9095-9102.

(32) Grimme, S.; Goerigk, L.; Fink, R. F. Spin-component-scaled electron correlation methods. Wiley Interdisciplinary Reviews: Computational Molecular Science 2012, 2, 886906.

(33) Krauter, C. M.; Pernpointner, M.; Dreuw, A. Application of the scaled-opposite-spin approximation to algebraic diagrammatic construction schemes of second order. The Journal of Chemical Physics 2013, 138, 044107.

(34) Mester, D.; Nagy, P. R.; Kállay, M. Reduced-cost second-order algebraic-diagrammatic construction method for excitation energies and transition moments. J. Chem. Phys. 2018, 148, 094111.

(35) Mester, D.; Kállay, M. Combined Density Functional and Algebraic-Diagrammatic Construction Approach for Accurate Excitation Energies and Transition Moments. Journal of Chemical Theory and Computation 2019, 15, 4440-4453, PMID: 31265275.

(36) MRCC, a quantum chemical program suite written by M. Kállay, P. R. Nagy, Z. Rolik, D. Mester, G. Samu, J. Csontos, J. Csóka, B. P. Szabó, L. Gyevi-Nagy, I. Ladjánszki, L. Szegedy, B. Ladóczki, K. Petrov, M. Farkas, P. D. Mezei, and B. Hégely. See also Z. Rolik, L. Szegedy, I. Ladjánszki, B. Ladóczki, and M. Kállay, J. Chem. Phys. 139, 094105 (2013), as well as: www.mrcc.hu.

(37) Hättig, C.; Weigend, F. CC2 excitation energy calculations on large molecules using 
the resolution of the identity approximation. The Journal of Chemical Physics 2000, $113,5154-5161$.

(38) Winter, N. O. C.; Hättig, C. Quartic scaling analytical gradients of scaled opposite-spin CC2. Chem. Phys. 2012, 401, 217.

(39) Matthews, D. A.; Stanton, J. F. A new approach to approximate equation-of-motion coupled cluster with triple excitations. J. Chem. Phys. 2016, 145, 124102.

(40) Szabados, A. Theoretical interpretation of Grimme's spin-component-scaled second order Møller-Plesset theory. J. Chem. Phys. 2006, 125, 214105. 
Figure 1: Potential energy curves following the gradient of low lying excited states of Methanimine relative to the ground state equilibrium energy (left panels) and their divergence from the respective CCSDT curve (right panels).
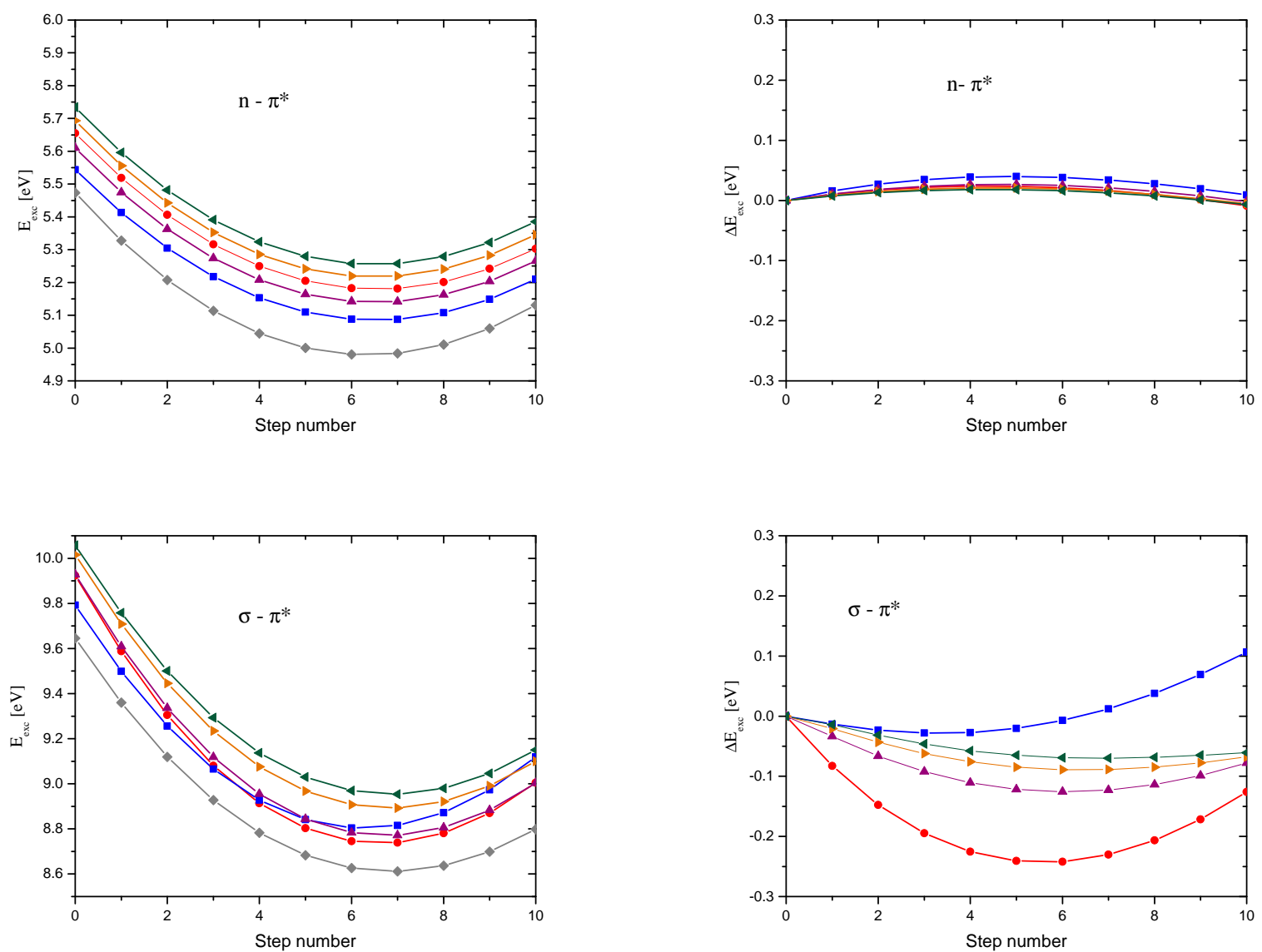

$$
\begin{aligned}
& -\bullet-C C 2 \\
& ---\operatorname{CCSD} \\
& ---\operatorname{SCS}-A D C(2) \\
& -\triangleleft-\operatorname{SOS}-A D C(2) \\
& ---\operatorname{ADC}(2) \\
& ---\operatorname{CCSDT}
\end{aligned}
$$


Figure 2: Potential energy curves following the gradient of low lying excited states of Formamide relative to the ground state equilibrium energy (left panels) and their divergence from the respective CCSDT curve (right panels).
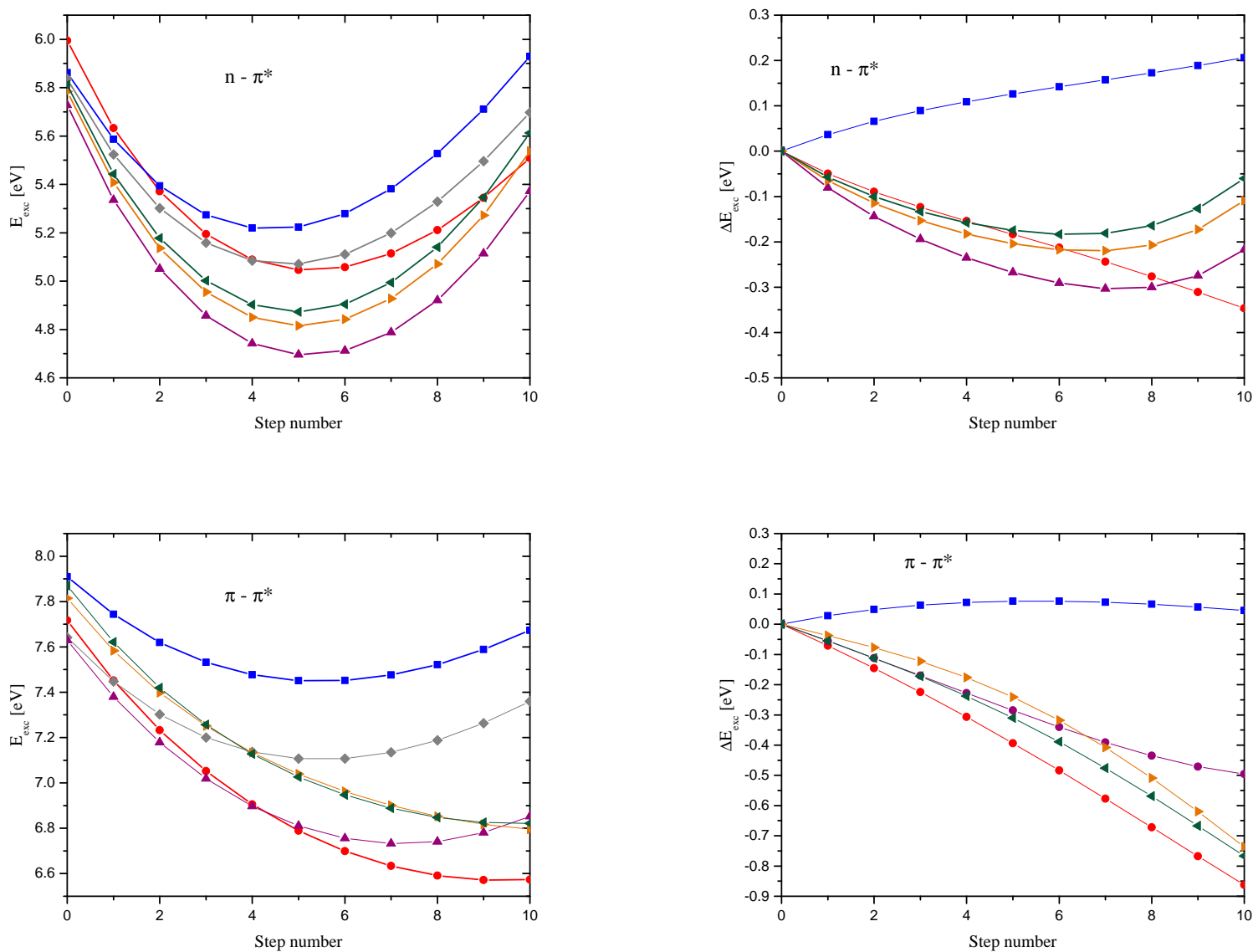

$$
\begin{aligned}
& -\bullet-\operatorname{CC} 2 \\
& -\square-\operatorname{CCSD} \\
& -\rightarrow-\operatorname{SCS}-A D C(2) \\
& -\triangleleft-\operatorname{SOS}-A D C(2) \\
& -\backsim-\operatorname{ADC}(2) \\
& -\bullet-\operatorname{CCSDT}
\end{aligned}
$$


Figure 3: Potential energy curves following the gradient of low lying excited states of Cytosine relative to the ground state equilibrium energy (left panels) and their divergence from the respective CCSDT curve (right panels).
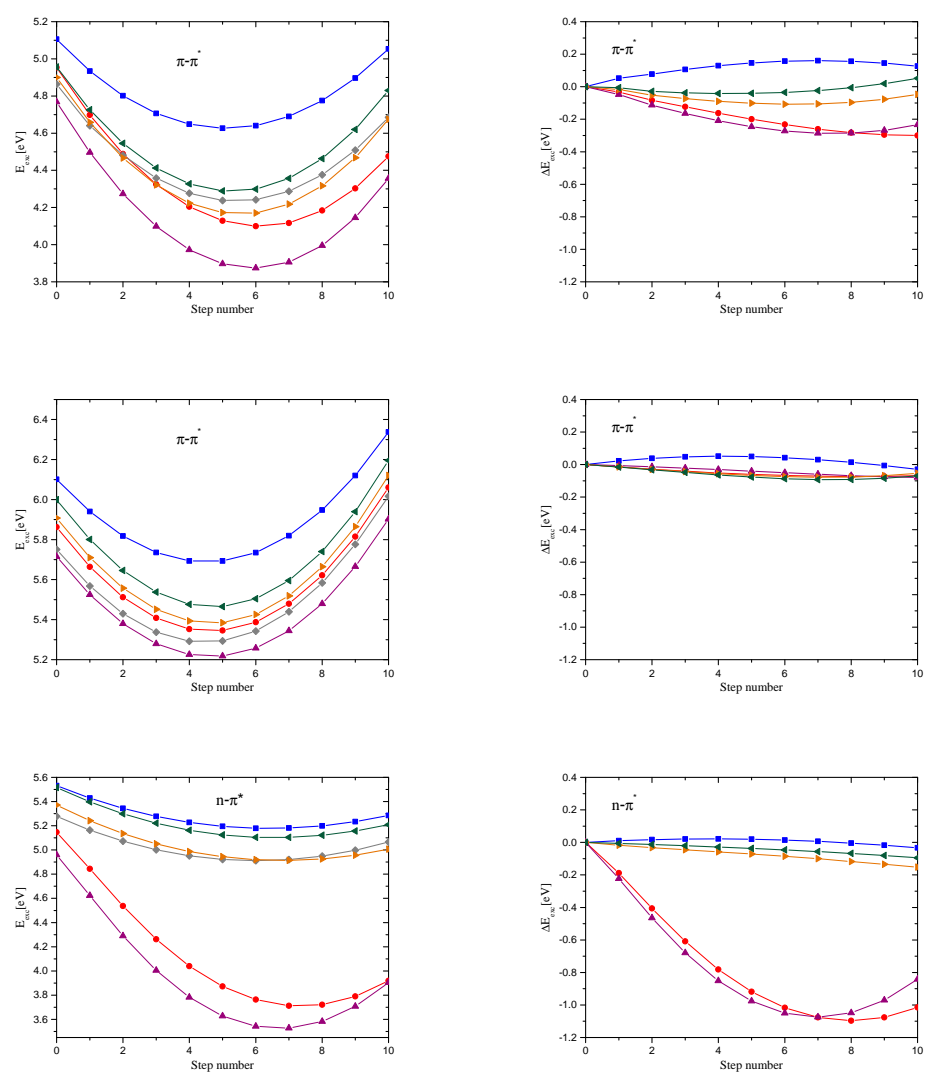

$$
\begin{aligned}
& -\bullet-\text { CC2 } \\
& ---\operatorname{CCSD} \\
& -\bullet-\operatorname{SCS}-A D C(2) \\
& -\triangleleft-\operatorname{SOS}-A D C(2) \\
& -\triangle-\operatorname{ADC}(2) \\
& -\bullet-\operatorname{CCSDT}
\end{aligned}
$$


Figure 4: Potential energy curves following the gradient of low lying excited states of Guanine relative to the ground state equilibrium energy (left panels) and their divergence from the respective $\operatorname{CCSD}(\mathrm{T})(\mathrm{a})^{*}$ curve (right panels).
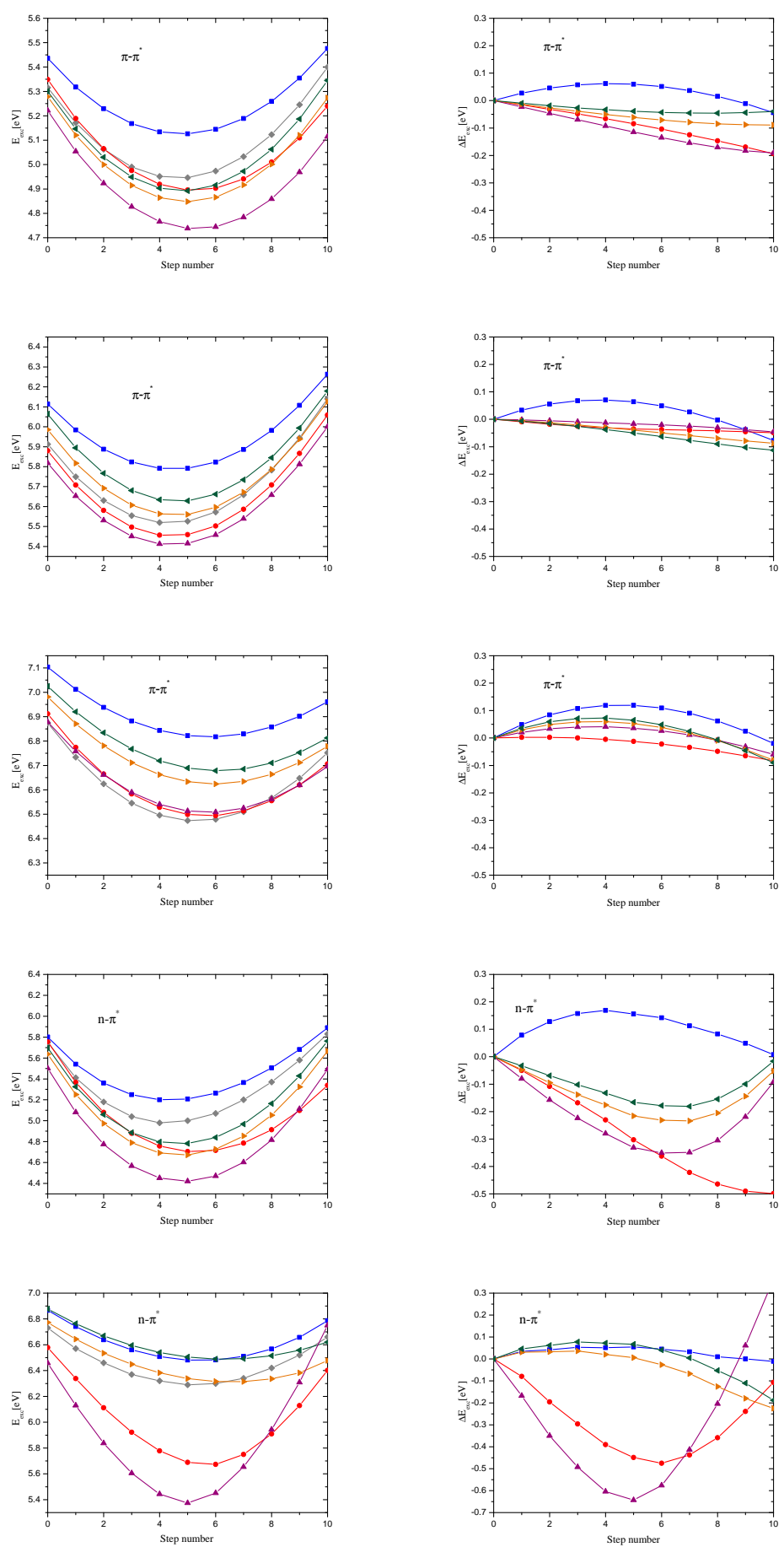

$$
\begin{aligned}
& -\bullet-\operatorname{CC} 2 \\
& ---\operatorname{CCSD} \\
& ---\operatorname{SCS}-A D C(2) \\
& -\triangleleft-\operatorname{SOS}-A D C(2) \\
& -\Delta-\operatorname{ADC}(2) \\
& --\operatorname{CCSD}(\mathrm{T})(\mathrm{a})^{*}
\end{aligned}
$$

